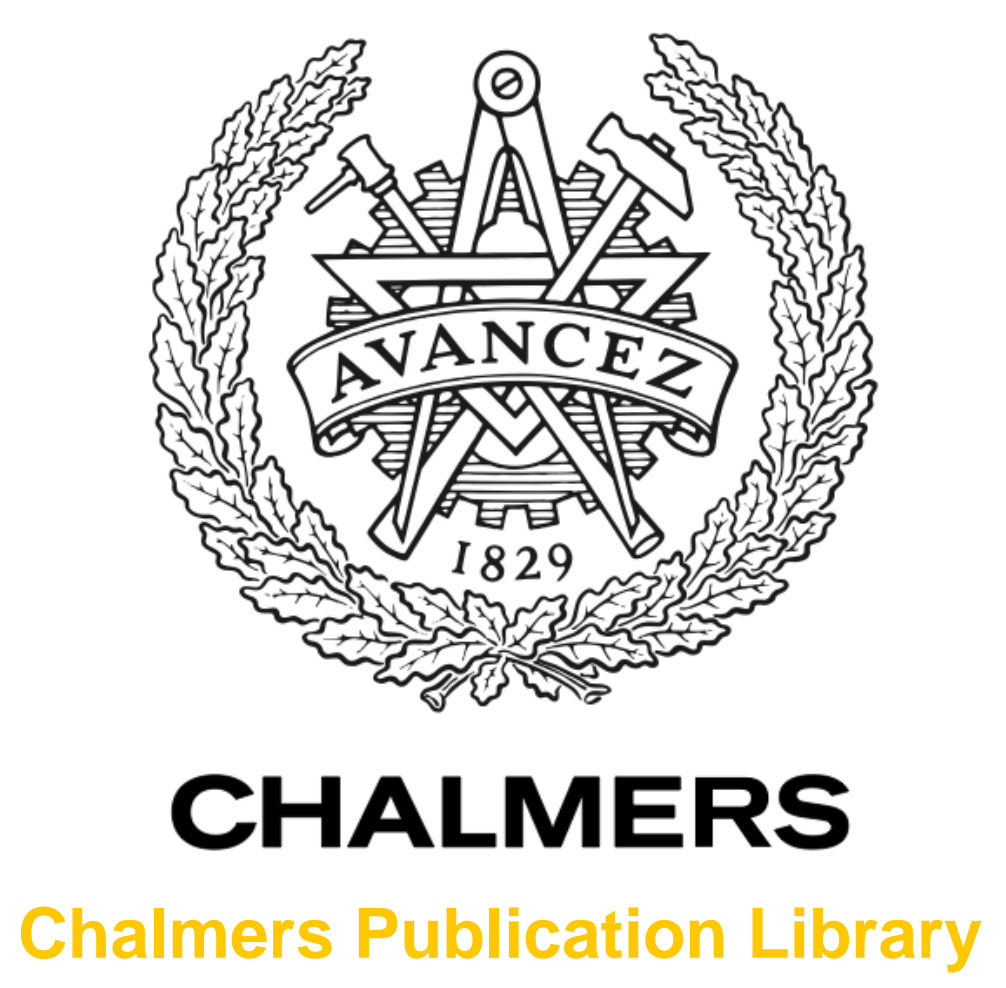

\title{
Towards a uniform evaluation of the science quality of SKA technology options: Polarimetrie aspects
}

This document has been downloaded from Chalmers Publication Library $(\mathrm{CPL})$. It is the author's version of a work that was accepted for publication in:

2012 14th International Conference on Electromagnetics in Advanced Applications, ICEAA 2012. Cape Town, 2 - 7 September 2012

Citation for the published paper:

Carozzi, T. ; Ivashina, M. (2012) "Towards a uniform evaluation of the science quality of SKA technology options: Polarimetrie aspects". 2012 14th International Conference on Electromagnetics in Advanced Applications, ICEAA 2012. Cape Town, 2 - 7 September 2012 pp. 543-546.

http://dx.doi.org/10.1109/ICEAA.2012.6328686

Downloaded from: http://publications.lib.chalmers.se/publication/166147

Notice: Changes introduced as a result of publishing processes such as copy-editing and formatting may not be reflected in this document. For a definitive version of this work, please refer to the published source. Please note that access to the published version might require a subscription. 


\title{
Towards a uniform evaluation of the science quality of SKA technology options: polarimetric aspects
}

\author{
T. D. Carozzi $^{1} \quad$ M. V. Ivashina ${ }^{2}$
}

\begin{abstract}
We discuss how to evaluate SKA technology options with regard to science output quality. In this work we will focus on polarimetry. We review the SKA specification for polarimetry and assess these requirements. In particular we will use as a illustrative case study a comparison of two dish types combined with two different feeds. The dish types we consider are optimized axi-symmetric prime-focus and offset Gregorian reflector systems; and the two feeds are the Eleven-feed (wideband) and a choked horn (octave band). To evaluate the imaging performance we employ end-to-end simulations in which given sky models are, in software, passed through a model of the telescope design according to its corresponding radio interferometrical measurement equation to produce simulated visibilities. The simulated visibilities are then used to generate simulated sky images. These simulated sky images are then compared to the input sky models and various figures-ofmerit for the imaging performance are computed. A difficulty is the vast parameter space for observing modes and configurations that exists even when the technology is fixed. However one can fixed certain standard benchmark observation modes that can be applied across the board to the various technology options. The importance of standardized, end-to-end simulations, such as the one presented here, is that they address the high-level science output from SKA as a whole rather than low-level specifications of its individual parts.
\end{abstract}

\section{INTRODUCTION}

The Square Kilometre Array (SKA) is an international project to construct a large radio telescope. SKA has numerous key science projects (KSP) that each have their own particular science requirements. The technology that will be used to achieve the various science goals has yet to be decided in detail. An important task within the SKA now is to assess the various proposed technologies for scientific potential with respect to the KSP objectives.

In this work we initiate an investigation in to how the proposed SKA technologies will perform scientifically by using simulations, and how to assess this performance in a concise yet meaningful way. It is important that a uniform methodology for performance evaluation is developed separately from the engineering consortia that design the SKA technology prototypes. Although standard performance criteria for radio telescopes exist, these are mainly at low-end, that is, parts of technology or engineering interfaces, which could easily be provided by the individual prototype developers themselves. What is still needed is a high-end, holistic assessment of the science performance of a fully assembled SKA design. To that end, one must rely on end-to-end simulations based on specifications provided by the prototype engineers. Such end-to-end simulations take sky map brightness models and pass them through the proposed SKA designs and produce simulated scientific data products (as per KSP).

In this paper we will limit ourselves to polarimetric aspects of science quality assessment, and in the near future we hope to consider other aspects.

\section{Review of polarimetric requirements of SKA}

The SKA is designed to be a fully polarimetric telescope, and several of its KSPs explicitly require full-polarization data products. As such they have provided a specification of the quality of the polarimetry required for the SKA. In the Table, an executive level specification of the polarization purity for some of the KSPs is provided. This Table is a gross simplification of the more detailed description provided in the Design Reference Mission report [2] and the preliminary SKA specification [3].

\begin{tabular}{|l|l|}
\hline \multicolumn{1}{|c|}{ KSP } & Polarization Purity [dB] \\
\hline EoR & -30 \\
\hline Cosmic Magnetism & -30 \\
\hline Pulsar timing & -40 \\
\hline
\end{tabular}

Table 1: Executive level requirements for some of the KSP on SKA polarization purity.

\section{Issues regarding the SKA polarization specification}

When referring to the polarimetric performance of the telescope, the SKA preliminary specifications use the term polarization purity. Although this quantity is not explicitly defined in the SKA documents, the units are given in negative decibels, and the implicit suggestion is that this is one of the four (unclear which) standard cross-polarization ratios (XPR) for dual-polarized feeds, see [1].

The SKA specification also mixes its use of raw polarization purity (in which no calibration has been

\footnotetext{
${ }^{1}$ Onsala Space Observatory, Chalmers University Sweden, e-mail: tobiad chalmers. se, tel.: +46 (0)31 7725518

${ }^{2}$ Chalmers University Sweden, e-mail: marianna. ivashina@chalmers . se, tel.: +46 (0)31 7721812.
} 
applied to data) and post-calibration polarization purity (in which knowledge of the polarization properties of the dual-polarized feed is applied to data). This makes it unclear whether it is the hardware or the software that should be optimized to achieve the specified polarization purity.

For these reasons we suggest that the SKA specification for polarization purity should be given in the recently introduced quantity called intrinsic cross-polarization ratio (IXR) defined in [1]. IXR is a unique property of the dual-polarized feed that sets the limits of its calibratability, which is a much more practically useful concept. It also impacts on the overall sensitivity of the feed.

\section{$3 \quad$ IXR requirements by KSP}

We now comment briefly on the polarimetirc quality requirements of some of the KSPs using the IXR quantity as a figure-of-merit for polarization purity.

\subsection{Epoch of Reionization}

The Epoch of Reionization KSP requires good polarimetry in particular to discern leakage from the polarized galactic foreground, see [4]. As EoR is mainly towards the lower end of the SKA frequency band, this is mainly a requirement on the aperture arrays (AA) that are envisaged to cover these frequencies. AAs are phased arrays of fixed mounted antennas, i.e. the AAs are not mechanically pointed, and so their polarimetric properties are a function of elevation and azimuth rather than offset angles relative to pointing direction.

It is important to note that AAs are different polarimetrically speaking from dishes. Firstly, they tend to be bind towards the horizon. This elevation effect is due to the fact that sources close to the horizon can have a large vertical electric field component, due to geometry, but AAs lack a vertical component receptor, their dual-polarized receptors being nominally in the the horizontal plane. For this reason the best polarimetry from AAs is expected to be around zenith, rather than along the (electronic) pointing direction. Secondly, they have a strong azimuthal variation depending on whether the source of interest is along the $\mathrm{E}$ - or $\mathrm{H}$-planes (i.e. aligned with antennas linear extent), or along the D-plane (i.e. diagonal to the crossed antennas).

\subsection{Cosmic magnetism}

The Cosmic magnetism KSP's main science goal is to produce rotation measure (RM) synthesized maps, which are computed from the Faraday rotation as a function of frequency of polarized background sources. This therefore requires high polarizedsignal sensitivity to clearly detect polarized sources. By contrast, instrumental polarization leakage has typically not been considered an issue in RM synthesis. This is because it has been assumed that the polarization leakage is constant in frequency or at least does not induce an apparent rotation with frequency. Under this assumption only the value of the Faraday spectrum at Faraday depth zero would be affected, which is a relatively uninteresting Faraday depth scientifically.

Recent unpublished results by one the authors (TC), however, indicate that even simple dipole-like crossed-antennas can exhibit a monotonic apparent rotation with frequency. This rotation is small, less than a full turn, over the frequency band of the dipoles, but nonetheless could be significant enough to be measurable in practice. For more complex antennas such dense AAs which are known to exhibit mutual-coupling, it is likely to expect a more complex variation of polarization rotation with frequency. Thus it is important for the SKA to assess this effect in the various proposed technologies, as it may have an impact on the quality of the scientific output of the Cosmic magnetism KSP. IXR variation as a function frequency is then a useful measure for the calibratability of RM synthesise.

\subsection{Pulsar timing}

Pulsar timing precision can be enhanced by using full-polarization information rather than just intensity profiles, see [5]. A simple explanation for the improvement is that polarization has more degrees of freedom to match pulsar profiles in time.

Recently one of the authors (TC), together with SKA pulsar scientists, have run pulsar timing simulations as a function of telescope IXR. The main preliminary result is that IXR values better than $-20 \mathrm{~dB}$ do not improve pulsar timing accuracy. If this result holds, then the polarization purity requirement for the pulsar KSP can be lowered considerably.

\section{Comparison of technologies with respect to polarimetric quality}

Here we present a case study comparison of two different feeds with regards to polarimetric quality. The results shown here are not meant as a definitive assessment of the performance of the various technologies, but rather as an illustration of the methodology that can be used for a proper assessment. The methodology we use is end-to-end simulation of radio interferometric imaging. In more detail, this means generating a mock sky brightness model and then computing the measured visibilities using the radio interferometric measurement equation [6]; then from these measured visibilities we compute a synthesized image.

In practice we have used the standard software package MeqTrees [7]. A 3x3 cartesian grid (with 30 ' spacing) of $1 \mathrm{Jy}$ unpolarized point sources was generated. We arranged a WSRT-like array of primefocus dishes fitted with either the Eleven-feed [8] or a horn-feed. The feeds were equatorially mounted, and we used actual, measured Jones matrices for the 
Eleven-feed and EMC simulated Jones matrices for the horn-feed. Then we ran a simulated observation of $11 \mathrm{~h}$ sampled every $15 \mathrm{~min}$. The measured visibilities were then treated as real observations and passed through the lwimager imaging software. Natural weighting was used and a Clark cleaning was applied. The motivation for this particular simulation setup was to realize a simple benchmark test with a minimal number of real-world interferometer effects to focus on the performance of the feeds.

In Figs 1-4, we show simulation results of polarimetric imaging with the Eleven-feed and a horn-feed at the prime-focus of $15 \mathrm{~m}$ dishes (f/D=0.42). The images were CLEAN-ed, and only the Stokes I (flux brightness) and Stokes V (circular flux brightness) are shown as the Stokes Q and U are vanishingly small. From the Stokes I images one can see that the Eleven-feed has a more uniform gain than the horn-feed over the field of view, which is about $0.5^{\circ}$. From the Stokes V images one can see that the Eleven-feed has a higher degree of instrumental polarization, as deduced from the fact that the true image was unpolarized but the synthesized CLEAN image shows residual polarization. The absolute value of the Stokes V brightness divided by the Stokes I is the degree of instrumental polarization which in turn is equal to the Mueller version of IXR (IXRM). Thus the IXRM for the horn-feed is approximately $-50 \mathrm{~dB}$ on-axis and $-30 \mathrm{~dB}$ off-axis along the D-planes $\left(45^{\circ}\right.$ diagonal directions) at the edge of the field, while for the Eleven-feed it is $-15 \mathrm{~dB}$ on-axis and $-13 \mathrm{~dB}$ along the D-planes at the edge of the field.

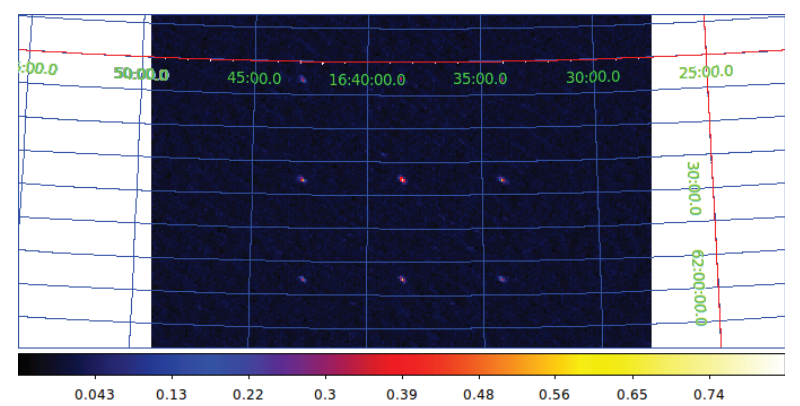

Fig 1 Simulated Stokes I image of a $3 \times 3$ grid of unpolarized 1 Jy sources with a spacing of 30' for an measured Eleven-feed in a prime-focus dish at $1.0 \mathrm{GHz}$. Color bar units in Jy.

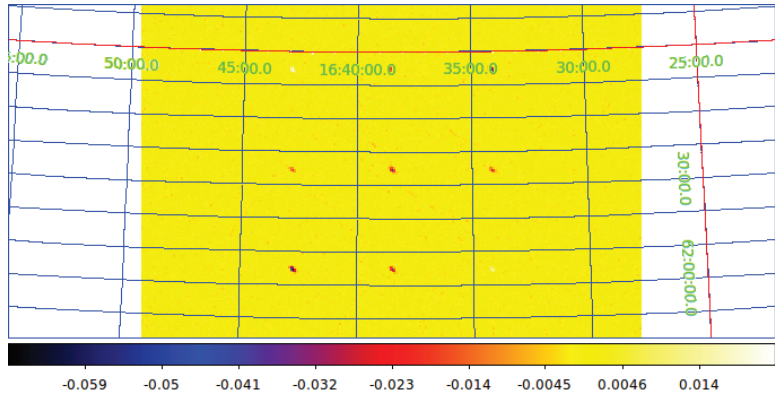

Fig 2 Same as in Fig. 1 but for Stokes V.

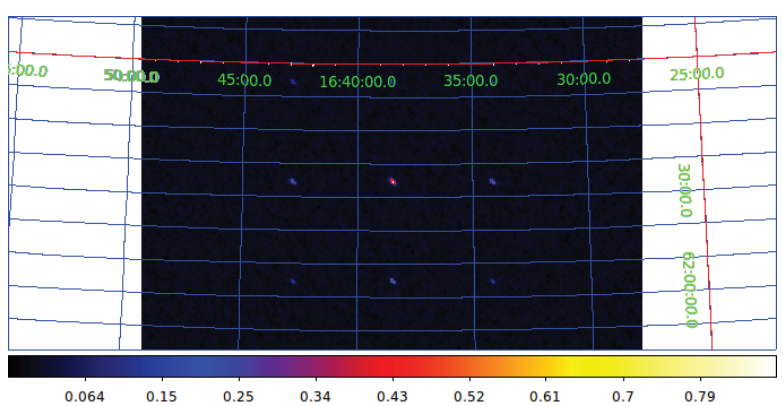

Fig 3 Same as in Fig 1 but for a EMC simulated hornfeed at $1.4 \mathrm{GHz}$.

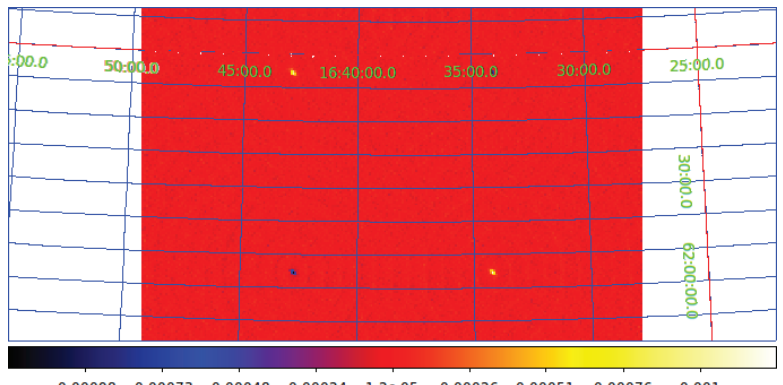

$\begin{array}{lllllllllll}0.00098 & 0.00073 & 0.00048 & 0.00024 & 120-05 & 0.00026 & 0.00051 & 0.00076 & 0.001\end{array}$

Fig 4 Same as in Fig 3 but for Stokes V.

The reason for the instrumental polarization in the Eleven-feed is related to limitations in the prototype used during measurements. In particular there was a difference between the gains of the power combiners used to feed the antenna, which was allowed since this prototype was not optimized for crosspolarization leakage. However the result illustrates that IXR is affected by differential gains and that this has a effect on science quality. Thus these results can be used to set differential tolerances on the LNAs and not just the antenna proper.

Again, the results shown here are not intended as a definitive assessment of the feed technologies, but rather as an illustration of the potential science level assessment of the entire signal chain in proposed SKA designs. It should be noted that feed 
comparison is not very meaningful as we used real gain patterns for the Eleven-feed and simulated gain patterns for the horn-feed.

\section{Conclusions}

In this work we have presented a unified approach to assessing the scientific performance of various SKA related technologies, in particular with respect to polarimetry. This will be of value in the process of selecting technologies for the SKA. We have implemented end-to-end simulations of the SKA and introduced science quality figure-of-merits.

We have considered the KSPs that are polarization drivers and analyzed their requirements on the basis of the newly conceived figure-of-merit for polarimetry called IXR. IXR is a unique XPR that sets the fundamental limit of the polarimetric performance of a polarimeter [1]. It can therefore be used in place of the four generic XPR values, and regardless of whether polarimetric calibration has or has not been applied.

We have also assessed the polarimetrical imaging performance of the Eleven-feed versus a horn-feed and found differences in gain uniformity and instrumental polarization over the field of view when they implemented in a simulated interferometer array.

Further work is needed to properly assess the full SKA.

\section{Acknowledgments}

The authors will like to acknowledge the joint work together with Aris Karastergiou on polarimetric pulsar timing.

\section{References}

[1] T. D. Carozzi and G. Woan, "A Fundamental Figure of Merit for Radio Polarimeters", IEEE trans. ant. prop., 59, 6, 2058, doi:10.1109/TAP.2011.2123862, 2011 .

[2] SKA science working group, "The Square Kilometre Array Design Reference Mission: SKA Phase 1", SKA project document, 2011.

[3] R. Schilizzi et al., "Preliminary Specification for the Square Kilometre Array", SKA memo series, 2007.

[4] V. Jelić et al., "Realistic simulations of the Galactic polarized foreground: consequences for $21-\mathrm{cm}$ reionization detection experiments", MNRAS, 409, 4, 2010

[5] J.M. Cordes, et al., "Pulsars as tools for fundamental physics and astrophysics", New Astronomy Reviews, 48, 11-12, 1413-1438, 2004.

[6] J.P. Hamaker, R.J. Sault and J.D. Bregman, "Understanding Radio Polarimetry:
I. Mathematical foundations", Astron. Astrophys. Suppl. Ser. 117, 137-147, 1996.

[7] J.E. Noordam and O.M. Smirnov, "The MeqTrees Software System And Its Use For Third-Generation Calibration Of Radio Interferometers", A\&A 524 A61, 2010.

[8] W.-Ch. Liao, M. V. Ivashina, P.-S. Kildal and A. van Ardenne, "Effects of Struts on the Performance of Axisymmetric Reflector Antenna Systems with Compact UWB 'Eleven Feed"', Proc. of the 6th European Conf. on Antennas and Propagation (EuCAP2011), Prague, 2012 